\title{
Hysteresis in rf-driven large-area josephson junctions
}

\author{
Olsen, O. H.; Samuelsen, Mogens Rugholm
}

Published in:

Physical Review B

Link to article, DOI:

10.1103/PhysRevB.34.3510

Publication date:

1986

Document Version

Publisher's PDF, also known as Version of record

Link back to DTU Orbit

Citation (APA):

Olsen, O. H., \& Samuelsen, M. R. (1986). Hysteresis in rf-driven large-area josephson junctions. Physical Review B, 34(5), 3510-3512. https://doi.org/10.1103/PhysRevB.34.3510

\section{General rights}

Copyright and moral rights for the publications made accessible in the public portal are retained by the authors and/or other copyright owners and it is a condition of accessing publications that users recognise and abide by the legal requirements associated with these rights.

- Users may download and print one copy of any publication from the public portal for the purpose of private study or research.

- You may not further distribute the material or use it for any profit-making activity or commercial gain

- You may freely distribute the URL identifying the publication in the public portal

If you believe that this document breaches copyright please contact us providing details, and we will remove access to the work immediately and investigate your claim. 


\title{
Hysteresis in rf-driven large-area Josephson junctions
}

\author{
O. H. Olsen \\ Systems Engineering, Niro Atomizer, Gladsaxevej 305, DK-2860 Soeborg, Denmark \\ M. R. Samuelsen \\ Physics Laboratory I, The Technical University of Denmark, DK-2800 Lyngby, Denmark
}

(Received 24 March 1986)

\begin{abstract}
We have studied the effect of an applied rf signal on the radiation emitted from a large-area Josephson junction by means of a model based on the sine-Gordon equation. The rms value of the voltage of the emitted signal has been calculated and a hysteresis loop found. An analysis shows that the hysteresis is due to the nonlinearity in the system, i.e., the dynamics of the lower branch can be described by a solution to the linearized system while the upper branch is described by a breather mode. These solutions are frequency locked to the driving signal. Various characteristics of the loop such as threshold value and level of the branch are predicted analytically.
\end{abstract}

The study of the dynamical behavior of large-area Josephson junctions is of fundamental as well as practical interest. On the one hand, the characteristics of Josephson junctions have a rich content of nonlinear properties which are suitable for detailed investigations of various areas such as nonlinear wave dynamics ${ }^{1,2}$ and chaotic states. ${ }^{3-7}$ Further, the system is suitable for testing of perturbation approaches. ${ }^{2,8}$ On the other hand, there have been suggestions for applications of large-area Josephson junctions within such diverse fields as microwave oscillators-amplifiers and data processing systems.

In most experiments in Josephson physics one finds hysteresis phenomena. Thus, an understanding of the basic dynamics behind the hysteresis is important. In the present paper we focus on large-area Josephson junctions (overlap tunnel junctions) irradiated by rf signals (without applied dc current). The microwave pump signal is applied to one end of the junctions. This system is modeled by a perturbed one-dimensional sine-Gordon equation with approximate boundary conditions describing the influence of the rf signal. Period doubling (i.e., the first steps in the transition to chaos) has been found for the model and in experiments in Refs. 6 and 7, respectively. We have examined this system by means of the model. In order to get an understanding of the influence of the applied rf signal on the emitted radiation we have calculated the rms value of the emitted voltage as a function of the amplitude of the applied signal.

This relation exhibits hysteresis. The dynamics describing the lower branch can be found linearizing the model equation while the dynamics of the upper branch can be well described as a breather mode frequency locked to the driving signal. Application of a perturbation approach gives expressions for the level and the lower threshold for the upper branch.

The dynamics of a long Josephson tunnel junction is assumed to be governed by a perturbed sine-Gordon equation

$$
\Phi_{x x}-\Phi_{t t}=\sin \Phi+\alpha \Phi_{t}
$$

where $\Phi$ is the phase difference between the two supercon- ducting films. The spatial variable is measured in units of the Josephson penetration depth $\lambda_{J}=\left(\hbar / 2 e d \mu_{0} J\right)^{1 / 2}$ and the time in units of the reciprocal plasma frequency $\omega_{0}^{-1}$, where $\omega_{0}=(2 e J / \hbar C)^{1 / 2}$. Here $J$ is the Josephson current density, $d$ is the magnetic thickness of the barrier, and $C$ is the capacitance per unit area. The loss $\alpha$ is defined through the relation $\alpha=G(\hbar / 2 e J C)^{1 / 2}$, where $G$ is the shunt conductance per unit area.

With an oscillating magnetic field $H_{e} \sin \omega t$ applied to one end of the junction, perpendicular to the length of the junction and parallel to the plane of the barrier, the boundary conditions for the phase difference $\Phi$ at the ends are

$$
\begin{aligned}
& \Phi_{x}(l)=a \sin \omega t, \\
& \Phi_{x}(0)=0,
\end{aligned}
$$

where $a=H_{e} / J \lambda_{J}$ and $l=L / \lambda_{J}$ are the normalized magnetic field strength and junction length, respectively.

Equations (1) and (2) have been solved numerically. In the numerical solution we have applied an approximation based on a stabilized leap-frog scheme. ${ }^{9}$ Throughout this paper the frequency $\omega=0.8$, the loss parameter $\alpha=0.2$, and the normalized length $l=5$ are held fixed. In Fig. 1 we show the rms value of $\Phi_{t}$ in the right end of the junction as a function of the amplitude $a$ resulting from numerical simulations. In order to simulate an experimental situation the curve has been obtained in the following way: For $a=0$ we use flat initial conditions [i.e., $\Phi(x, 0)=0$ and $\Phi_{t}(x, 0)=0$ ], $a$ is then increased gradually by an amount $\Delta a$ (using a ramp function $a_{\text {new }}=a_{\text {old }}+(\Delta a / 50) t$ for the time $t \leq 50$ ). When steady state is obtained the computations are stopped, restarted (the initial conditions now being the former obtained steady solution), and $a$ gradually increased. This procedure was continued for $a<0.8$ with $\Delta a=0.1$, while for $a>0.8, \Delta a=0.05$. The steady state was typically obtained after $t=500$ and each run has typically been continued to $t=2000$.

In Fig. 1 it is seen that the rms value changes rapidly for $a_{\mathrm{u}, \mathrm{th}} \approx 0.87$ and saturates at an almost constant level (referred to as the upper branch). This change corresponds 


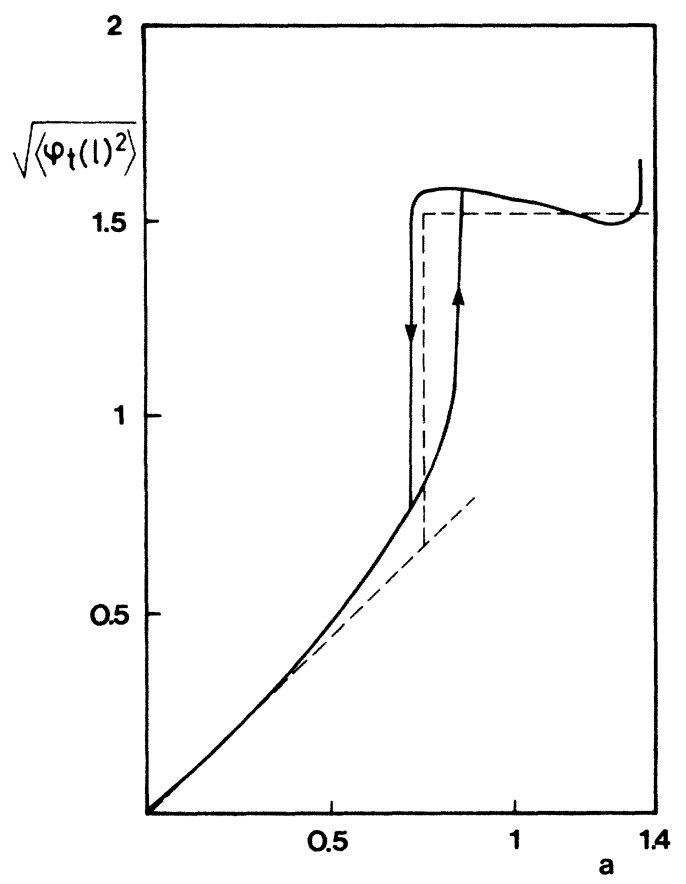

FIG. 1. The rms value $\left(\left\langle\Phi_{l}^{2}\right\rangle\right)^{1 / 2}$ vs $a$. Full lines indicate numerical results-broken lines indicate results obtained by approximations.

to a change in the wave dynamics. The saturation level keeps almost constant up to $a \approx 1.35$. $^{10}$ From $a=1.3$ we start reducing $a$ and find that the rms value decreases rapidly and returns to the lower branch at a value $a_{1, \text { th }} \approx 0.74$ $<a_{\mathrm{u}, \mathrm{th}}$. In the following we explain this hysteresis loop by identifying the modes sustaining the lower and upper branch, respectively. An approximate solution to Eqs. (1) and (2) was found in Ref. 6 for $a \ll 1$ (or $a \gg 1$ ) by linearizing the model. The solution is

$$
\Phi(x, t)=-a \exp (-i \omega t) \cos k x /(k \sin k l),
$$

where $k$ (complex) is given by the dispersion relation

$$
k^{2}=\omega^{2}-1+i \alpha \omega=|k|^{2} \exp (i 2 \theta) \text {, }
$$

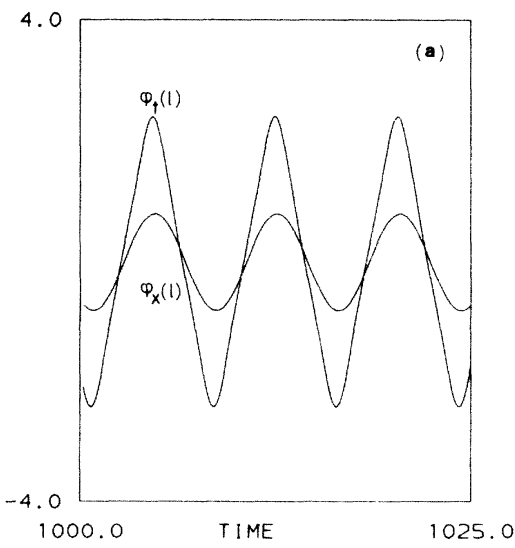

with

$$
|k|=\left[\left(\omega^{2}-1\right)^{2}+\alpha^{2} \omega^{2}\right]^{1 / 4}, \tan 2 \theta=\frac{\alpha \omega}{\omega^{2}-1} .
$$

The rms value of $\Phi_{t}$ for this solution is given by

$$
\begin{aligned}
\left(\left\langle\Phi_{t}^{2}\right\rangle\right)^{1 / 2}= & \frac{a \omega}{2^{1 / 2}|k|} \\
& \times\left(\frac{\cosh (2|k| l \sin \theta)+\cos (2|k| l \cos \theta)}{\cosh (2|k| l \sin \theta)-\cos (2|k| l \cos \theta)}\right)^{1 / 2} .
\end{aligned}
$$

The lower broken line in Fig. 1 is this expression which shows that the mode sustaining the lower branch is well described by the solution to the linear system.

The mode sustaining the upper branch has been identified as a breather frequency locked to the driver signal and placed symmetrically with respect to the right end of the junction $(x=l)$. Thus the mode describing the upper branch is given by the stationary breather solution

$$
\Phi(x, t)=4 \tan ^{-1}\left(\frac{\left(1-\omega^{2}\right)^{1 / 2}}{\omega} \frac{\sin \left(\omega t+\theta_{\mathrm{br}}\right)}{\cosh \left[\left(1-\omega^{2}\right)^{1 / 2}(x-l)\right]}\right) .
$$

We have compared this expression displayed in a phase plane to the numerical solution of Eqs. (1) and (2) and found an agreement within a few percent. Using this expression we get

$$
\left\langle\Phi_{t}(l, t)^{2}\right\rangle=\frac{\omega}{2 \pi} \int_{0}^{2 \pi / \omega} \Phi_{t}^{2}(l, t) d t=8 \omega\left(1-\omega^{2}\right) .
$$

From Eq. (7) we find the upper broken line in Fig. 1 which models the saturation level. The agreement between the numerical results and the analysis is good. In order to determine an expression for the lower threshold value $a_{1, \text { th }}$ for the saturation level we apply the usual perturbation approach. ${ }^{11}$ Defining the energy,

$$
H=\int_{0}^{l}\left[\frac{1}{2} \Phi_{x}^{2}+\frac{1}{2} \Phi_{t}^{2}+(1-\cos \Phi)\right] d x .
$$

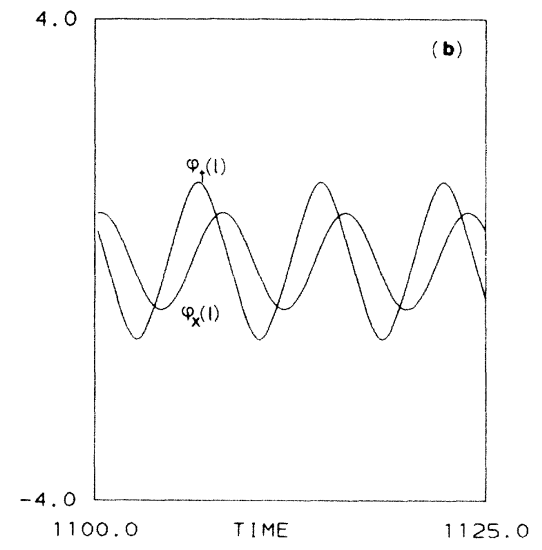

FIG. 2. Voltage $\Phi_{t}(l, t)$ and applied rf signal $\Phi_{x}(l, t)$ vs time for $a=0.8$. The steady-state solution on the upper branch (a) and lower branch (b). The curves of smaller amplitude are the applied rf signal. 
Time differentiation of $H$ and use of Eqs. (1) and (2) yield

$$
\frac{d H}{d t}=-\alpha \int_{0}^{l} \Phi_{t}^{2} d x+\left.\Phi_{x} \Phi_{t}\right|_{0} ^{l}
$$

In this equation the first and second term represent energy dissipation and energy input, respectively. The energy is dissipated in the whole junction while energy is induced through the external field.

The energy change in one period of oscillation is

$\Delta H=-\alpha \int_{0}^{2 \pi / \omega} \int_{0}^{l} \Phi_{t}^{2} d x d t+\left.\int_{0}^{2 \pi / \omega} \Phi_{x} \Phi_{t}\right|_{x=l} d t$

Now, in a stationary situation this change in energy is zero. Close to the threshold value the energy input is at a maximum forcing the phase angle $\theta_{\mathrm{br}}=0$ in Eq. (6).

Inserting the breather solution in the right side of Eq. (10) yields $-16 \pi \alpha \sin ^{-1}\left(1-\omega^{2}\right)^{1 / 2}$ for the first term where we have neglected the influence of the left boundary on the breather. The second term is evaluated as $8 \pi a[(1-\omega) /(1+\omega)]^{1 / 2}$ using Eq. (2) and the breather solution. Thus Eq. (10) can be written

$\Delta H=-16 \pi \alpha \sin ^{-1}\left(1-\omega^{2}\right)^{1 / 2}+8 \pi a\left(\frac{1-\omega}{1+\omega}\right)^{1 / 2}$.

Setting $\Delta H=0$ in this equation gives an expression for the lower threshold value

$$
a_{1, \mathrm{th}}=2 \alpha\left(\frac{1+\omega}{1-\omega}\right)^{1 / 2} \sin ^{-1}\left(1-\omega^{2}\right)^{1 / 2}
$$

For the parameter values used in this paper Eq. (12) yields $a_{1, \text { th }}=0.768$ - this is the vertical broken line in Fig. 1. The agreement is seen to be very good.

Finally, we present numerical results which illustrate
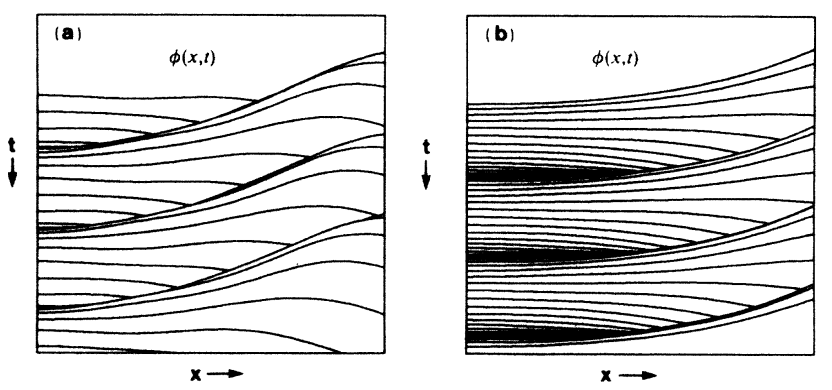

FIG. 3. Spatial variation of the phase difference $\Phi(x, t)$ for $a=0.8$ - upper branch (a) and lower branch (b). Time intervals as in Fig. 2.

the modes describing the dynamics on the branches. In Fig. 2 we have shown the voltage $\Phi_{t}(l, t)$ versus time on the upper (a) and lower (b) branch for $a=0.8$. For reference we have also displayed the applied $\mathrm{rf} \operatorname{signal}, \Phi_{x}(l, t)$ being the curves of smaller amplitude. In accordance with the analysis we see that the phase difference between $\Phi_{t}$ and $\Phi_{x}$ is very small in Fig. 2(a). In Fig. 3 the spatial variation of the modes is displayed in terms of $\Phi$ for the same time intervals as in the former figures.

In summary, our studies have shown that hysteresis in the investigated system is indeed a nonlinear phenomenon. The present work presents direct identification of the modes sustaining the branches in the hysteresis loop. Further, a simple perturbation analysis based on energy flow is used to predict various characteristics of this loop. We note that a variety of other interesting phenomena such as intermittency-type chaos and period doubling transition are known to exist in this system.
${ }^{1}$ D. W. McLaughlin and A. C. Scott, Phys. Rev. A 18, 1652 (1978).

2P. S. Lomdahl, Ph.D. thesis, The Technical University of Denmark, 1982 (unpublished); P. S. Lomdahl, O. H. Sørensen, and P. L. Christiansen, Phys. Rev. B 25, 5737 (1982).

${ }^{3}$ A. R. Bishop, K. Fesser, P. S. Lomdahl, and S. E. Trullinger, Physica D 7, 259 (1983).

${ }^{4}$ A. R. Bishop, K. Fesser, P. S. Lomdahl, W. C. Kerr, M. B. Williams, and S. E. Trullinger, Phys. Rev. Lett. 50, 1095 (1983).

5 J. C. Eilbeck, P. S. Lomdahl, and A. C. Newell, Phys. Lett. 87A, 1 (1981)
${ }^{6}$ O. H. Olsen and M. R. Samuelsen, Appl. Phys. Lett. 47, 1007 (1985).

${ }^{7}$ Yu. Ya. Divin, J. B. Hansen, J. Mygind, O. H. Olsen, N. F. Pedersen, and M. R. Samuelsen (unpublished).

${ }^{8}$ N. F. Pedersen and D. Welner, Phys. Rev. B 29, 2551 (1984).

${ }^{9}$ R. K. Dodd, J. C. Eilbeck, J. D. Gibbon, and H. C. Morris, Solitons and Nonlinear Wave Equations (Academic, London, 1982), p. 581.

${ }^{10}$ At $a \approx 1.3675$ intermittency-type chaos is observed.

${ }^{11}$ P. S. Lomdahl and M. R. Samuelsen, Phys. Rev. A 34, 664 (1986). 